\title{
Ein begeisterter Forscher und ein liebenswürdiger Mensch
}

\author{
Der Transplantationsimmunologe Fritz Bach starb in den USA im Alter von 77 Jahren
}

In den späten 60er Jahren gelang ihm mit einer speziellen Technik die Optimierung der Spenderauswahl für Knochenmarkspender. Seine immunologischen Forschungserkenntnisse führten zu einer rasanten Verbesserung der Transplantationsergebnisse. Er war einer der Pioniere in der Untersuchung von Möglichkeiten tierische Organe so zu konditionieren, dass der Mensch sie bei einer Transplantation nicht abstoßen würde - und letztlich ein Warner vor einer frühzeitigen Anwendung dieser Methode. Seine Neugierde und wissenschaftliche Brillanz verknüpfte er mit einer ebenso begeisterten und ansteckenden Lebensfreude und kulturellen Offenheit. Am 14. August ist der gebürtige Österreicher und langjährige Harvard Professor Fritz Bach mit 77 Jahren in den USA gestorben.

Die Technik, die Fritz Bach zur bestmöglichen Spender-Empfänger-Übereinstimmung entwickelte, bestand in der systematischen Vermischung von Zellen des Empfängers mit jenen des potentiellen Spenders, bis er jenen Spender fand, dessen Zellen keine Abwehrreaktion auf jene des Empfängers zeigte. Damit ließ sich vorab feststellen, ob die Spender und Empfänger kompatibel sind. Zum ersten Mal wurde diese Vorgangsweise 1968 eingesetzt: Von Dr. Robert A. Good, dem „Vater der Immunologie", bei einer Knochenmarktransplantation eines fünf Monate alten Buben. Fritz Bach selbst führte ein Team, das einen zweijährigen Buben mit einer Blutungsstörung und wiederholten Infektionen operierte. Einige Jahre später gelang es Bach das Verfahren zu beschleunigen. Anstatt nach Tagen, erhielt man nun nach Stunden das Ergebnis und konnte so das Instrument auch für die Spender-Empfänger-Kompatibilität bei Nierentransplantationen einsetzen. Darüber hinaus beschrieb er, wie die weißen Blutzellen klassifiziert und eingefroren werden können, um bei vielen potentiellen Spendern als Screening verwendet zu werden. Seine Arbeiten zur Kompatibilität von Spendern führten letztlich auch zu Experimenten, die die Identifikation des Major Histocompatibility Complex ermöglichten, ein System von Oberflächenproteinen, das die einzigartige Immunität jedes Menschen

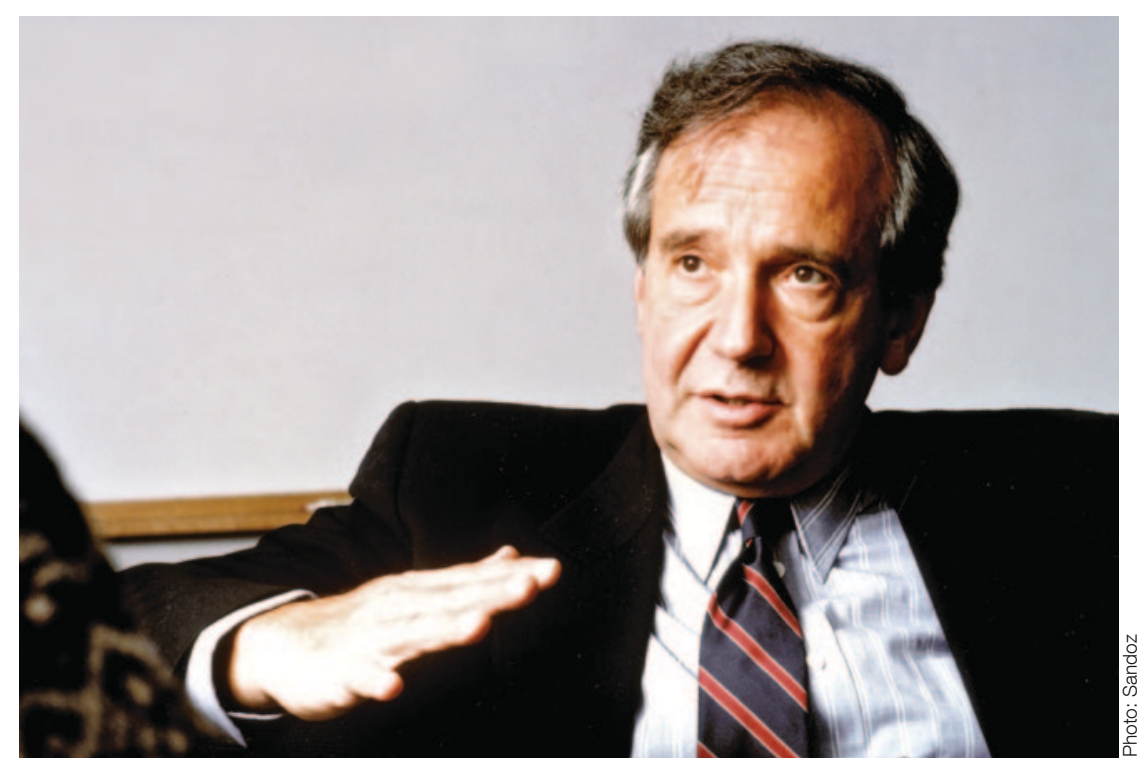

Fritz Bach - ein begeisterter und begeisternder Forscher und Humanist.

definiert. Seine Arbeiten über die Immunität und Abstoßungsreaktionen führten ihn in den 90er Jahren auch in das Feld der Xenotransplantation. Allerdings warnte er nachdrücklich vor den möglichen Gefahren, die durch eine Krankheitsübertragung von einem tierischen Organ auf den Menschen entstehen könnten. Seine neuesten Forschungen beschäftigten sich mit der Vermeidung von lebenslanger immunsuppressiver Medikation nach Transplantationen durch eine bessere Transplantattoleranz und er fand Hinweise, dass sehr niedrige Konzentrationen von inhaliertem Kohlenmonoxid helfen könnten, den Schaden von zerstörten Arterien zu minimieren.

In einer jüdischen Familie am 5. April 1934 geboren, wurde Fritz Bach mit seinem um zwei Jahre älteren Bruder 1939 nach der Reichskristallnacht nach England verschickt, wo ihn eine britische Familie aufnahm. Mit der eigenen Familie trafen die beiden Kinder später in Bath zusammen und sie emigrierten 1948 mit finanzieller Unterstützung eines amerikanischen Soldaten nach Amerika. Schule, Universität und Medizinische Universität absolvierte er in den USA in Burlington, Vermont und in Harvard und unterrichtetet schließlich selbst an der Universität von Wisconsin, an der Universität von Minnesota und kehrte nach Harvard zurück. 2004 erhielt Fritz Bach die österreichische Staatsbürgerschaft zurück und 2005 wurde er Ehrendoktor der Universität Wien, wo er auch ein Forschungslaboratorium gründete.

\section{„Fritz Bach war wahrschein-} lich auch der charismatischste Mensch, den ich je getroffen habe." Leo Otterbein

„Die Beiträge von Fritz Bach veränderten mehr oder weniger die Immunologie, wie wir sie kannten", zitierte The Boston Globe Leo Otterbein, einen Professor der Harvard Medical School: „Er war wahrscheinlich auch der charismatischste Mensch, den ich je getroffen habe und es war ein großes Vergnügen ihn um sich zu haben und immer sehr unterhaltsam." Seinen Humor offenbarte Fritz Bach auch in der Beschreibung seines Hauses in Manchester-by-the-Sea: „Das Haus liegt am Strand am Atlantischen Ozean und die Aussicht und der Klang und des Ozeans bewahren mich wahrscheinlich vor den Rechnungen eines Vollzeitpsychiaters." 